\title{
THE IMPACT OF FRAILTY ON COVID-19 OUTCOMES: A SYSTEMATIC REVIEW AND META-ANALYSIS OF 16 COHORT STUDIES
}

\author{
Y. YANG ${ }^{1, *}$, K. LUO ${ }^{2, *}$, Y. JIANG ${ }^{2, *}$, Q. YU ${ }^{3}$, X. HUANG ${ }^{2}$, J. WANG ${ }^{2}$, N. LIU ${ }^{4, \#}$, P. HUANG ${ }^{5, \#}$ \\ 1. Department of Neurology, Fifth People's Hospital of Chengdu, Chengdu, China; 2. Department of medicine, JingGangshan University, Ji' an,Jiangxi province, China; 3. College of \\ Nursing, Gannan Medical University,Ganzhou, Jiangxi province, China; 4. Department of neurology, the first affiliated hospital of Gannan medical university, Ganzhou, Jiangxi province, \\ China; 5. College of Nursing, Wenzhou Medical University, Wenzhou, Zhejiang province, China. * These authors contributed equally; \#They are co-first corresponding authors. \\ Corresponding author: Nanhai Liu, Department of neurology, the first affiliated hospital of Gannan medical university,Ganzhou, Jiangxi province, China. Email: 13970781916@163.com; \\ Pan Huang, College of Nursing, Wenzhou Medical University, Wenzhou, Zhejiang province, China. Email: gnyxywthp@163.com
}

\begin{abstract}
Background: Frail patients are increasingly vulnerable to stress, which is mainly manifested by a reduced physiologic reserve in metabolic and immune systems and neuromuscular system. Several studies found a significant association of frailty with COVID-19 severity to support the evidence for the application of frailty assessment. However, there were contradictory results in other studies. Thus we conducted a systematic review and meta-analysis to synthesize the current studies to investigate impact of frailty on COVID-19 outcomes and provide evidence-based decisions in clinical practice. Objective: We aimed to synthesize the current studies to investigate impact of frailty on COVID-19 outcomes and provide evidence-based decisions in clinical practice. Design: A systematic review and Meta-analysis of 16 cohort studies. Participants: Patients with COVID-19. Methods: A systematic retrieving for potential literature was conducted in several public electronic databases, including Medline(OvidSP), EMBASE, Pubmed and Chinese databases(China National Knowledge Infrastructure,Wanfang and Weipu) on August 1, 2020.The literature research was updated on October 26, 2020. Newcastle Ottawa Scale for cohort studies was used for quality assessment. RevMan (Version 5.3) and Stata 14.0 were used to synthesize the pooled effects. Results: According to the predefined inclusion and exclusion criteria, sixteen studies of 4324 patients were included in the final analysis. Frailty was significantly associated with increased risk of all-cause mortality among patients with COVID-19, with pooled adjusted odds ratios of 1.81 (95\% confidence intervals:1.48,2.21, I2=87.0\%, $\mathrm{P}<0.001)$. The result was consistent in stratified analysis to according to age, patient source, definitions of frailty, study quality, and adjustment method. Frailty was significant associated with an increased risk of COVID-19 severity, admission to intensive care unit, application of invasive mechanical ventilation, long-length stay. Conclusions: In this meta-analysis, we found frailty was significantly associated with an increased risk of clinical adverse events (all- cause mortality, COVID-19 severity, admission to the intensive care unit, application of invasive mechanical ventilation, long-length stay). Given the epidemic of COVID-19 and shortage of medical resources, paying more attention to screening frailty would contribute to disease management and resource allocation among patients with COVID-19.
\end{abstract}

Key words: COVID-19, frailty, meta-analysis, SARS-COV-2.

\section{Background}

The ongoing pandemic of coronavirus disease 2019 (COVID-19) caused by severe acute respiratory syndrome coronavirus 2 (SARS-CoV-2) has been a great threaten to public health worldwide (1). More than 76 million cases were diagnosed and 1.6 million died up to December 22, 2020 (2). As the medical shortage in the context of COVID-19, a number of studies aimed to construct risk factor models to achieve effective management of COVID-19 (3-5). In these models, age was a common parameter, and increasing age was significantly associated with adverse clinical outcomes $(6,7)$.

Frailty is a common syndrome with a decline in physiology, psychology and cognition (8). Frail patients are increasingly vulnerable to stress, mainly manifested by a reduced physiologic reserve in metabolic, immune and neuromuscular systems (9). Thus, frailty increases the risk of falls, disability, institutionalization and mortality (10-12). Although frailty is commonly used in old adults with a prevalence of $7.4 \%$ in elderly dwelling in community (13) and 52.3\% in nursing home (14), it is not limited to the old people. Muscedere et al found Received December 26, 2020

Accepted for publication January 12, 2021 that frailty was associated with 1.71-fold of hospital mortality compared with non-frail among patients in intensive care unit, which indicated a routine assessment of frail patients would contribute to high survival and recovery rate in intensive care unit (11). Additionally, it has been advocated by research that add frailty to the clinical assessment in patients with general surgery, acute coronary syndrome, and heart failure (15-20). In UK, the National Institute of Clinical Excellence recommend using the clinical frailty score to screen patients who need admission to intensive care units (21).

As COVID-19 is continuing to rage around the world and there is a lack of effective drugs to control the diseases, more attention should be paid to effective management for diseases to achieve the reasonable distribution of resources when the medical environment is under the tremendous pressure of medical shortage. Frailty was a concise and feasible tool used to screen patients at high risk of worsen outcomes. clinical frailty score was the most commonly used measurement tool, which mainly assessed the daily function of patients. Patients were categorized into nine levels from very fit (level one) to terminally ill (level nine). Frailty was determined when clinical
Published online March 4, 2021, http:/ / dx.doi.org/10.1007/s12603-021-1611-9 


\section{THE JOURNAL OF NUTRITION, HEALTH \& AGING}

frailty score great than 4 and pre-frailty as clinical frailty score great than (22-24). Several studies found a significant association of frailty with COVID-19 severity to support the evidence for application of frailty assessment (25). A multicentral retrospective study of ten hospitals found that clinical frailty score 7-9 was significant associated with 3.12-fold of seven-day all-cause mortality in comparison with clinical frailty score1-2 (26). In addition, Chinnadurai et al. demonstrated frailty was a key predictor factor for mortality among COVID19 patients hospitalized in an acute medical unit of secondary care level (27). However, Knopp et al reported that increased clinical frailty score was not associated with mortality risk with hazard ratio of 1.02 (95\% 0.93-1.12) (28). Another study demonstrated frailty was not useful for guiding clinical decision-making with an adjusted odds ratio of 1.6 (95\% 0.7$3.8)$ for clinical frailty score $4,1.6$ (95\% 0.7-3.9) for clinical frailty score 5 (29). In order to resolve the contradictory dispute and make clear the role of frailty in the context of the COVID19 epidemic, we systematically collected the current evidence and conducted a meta-analysis to investigate the impact of frailty on COVID-19 outcomes and provide evidence-based decisions in clinical practice.

\section{Methods}

The present study was conducted according to the Metaanalysis Of Observational Studies in Epidemiology (MOOSE) guidelines (see Appendix for Moose checklist).

\section{Search strategy and selection criteria}

A systematic retrieving for potential literature were conducted in several public electronic databases, including Medline (OvidSP), EMBASE, Pubmed and Chinese databases (China National Knowledge Infrastructure,Wanfang and Weipu) on August 1, 2020. The literature research was updated on October 26, 2020 (see Appendix for Search strategy). The search strategy was performed by combinations of MeSH terms (frailty and COVID-19) and keywords such as frailty (frail elderly), and COVID-19 (SARS-COV-2). We searched the potential gray studies by reviewing references of eligible articles.

\section{Study selection}

Two reviewers (Ying Yang and Kai Luo) blindly and independently screened retrieved studies. Inconsistencies between reviewers(whether a study should be included or not) were further discussed until consensus was reached.

\section{Inclusion and exclusion criteria}

The inclusion criteria are following: (i) studies analyzing the prevalence of frailty or the association of frailty with clinical events among patients with COVID-19. Exclusion criteria are as follows: (i)review and letter, (ii) case report and crosssectional studies, (iii) studies with insufficient data, (iv) studies in other language except for English and Chinese,(v) studies without a clear definition of frailty.

\section{Data extraction}

Two reviewers (Ying Yang and Jianghua Zhou) extracted the data from the included studies, including region, age, sample, study design, percentage of male, the source of patients, definition of frailty, outcome. The reviewers cross-checked all extracted data. Disagreements were discussed to reach a consensus.

\section{Assessment of risk of bias}

The quality of the included studies was assessed by using the Newcastle Ottawa Scale. Two reviewers performed quality assessment independently and blindly. The items of Newcastle Ottawa Scale for cohort studies were as following: (i) representativeness of the exposed participants; (ii) selection of non-exposed participants; (iii) ascertainment of exposed factors; (iv) no occurrence of outcomes of interest at baseline; (v) comparability; (vi) outcome assessment; (vii)enough period of follow up for outcomes to occur; and (viii) adequacy of follow up of cohorts.

\section{Study outcome definition}

Our present study aims to investigate the pooled prevalence and outcomes of frailty among patients with COVID-19. The outcomes included all-cause mortality, COVID-19 severity, admission to the intensive care unit, application of invasive mechanical ventilation, long-length $\operatorname{stay}(>10$ days).Patients were diagnosed as COVID-19 severity when they meet any of the following criteria: significant increase of respiration rate; hypoxia; consciousness disorder; food refusal or feeding difficulty and dehydration; other manifestations, such as coagulation disorders, cardiac damage, gastrointestinal disorder, increased level of liver enzyme, and rhabdomyolysis; critically ill cases (30).

\section{Statistical analysis}

The risk ratio was used as the common measure of association between frailty and all-cause mortality across studies.In order to synthesize the pooled effects, odds ratio was transformed into risk ratio using the formula risk ratio $=$ odds ratio/[(1- $\mathrm{P} 0)+(\mathrm{P} 0 \mathrm{x}$ odds ratio $)]$, and hazard ratio was considered as risk ratio $(31,32)$. When the exact association measures were impossible to be extracted from the reports, we recalculated the odds ratio and its variance was recalculated according to standard methods.The software RevMan (Version 5.3) and Stata 14.0 were used for meta-analysis analysis.To investigate the association between frailty and mortality risk, the risk ratio and $95 \%$ confidence interval were calculated. Fixed-effect and random-effect meta-analysis were conducted using the Mantel-Haenszel method. The chi-square test for Cochrane's Q statistic and I2 were used tp assess heterogeneity. When I2 was more than 50\%, heterogeneity existed, and 
THE IMPACT OF FRAILTY ON COVID-19 OUTCOMES

Table 1

Characteristics of included studies

\begin{tabular}{|c|c|c|c|c|c|c|c|c|}
\hline Study & Region & Age & Sample & Study design & Male & Patients type & Definition of frailty & Outcome \\
\hline $\begin{array}{l}\text { Chinnadurai R } 2020 \\
\text { (27) }\end{array}$ & England & $74 \pm 4.5$ & 215 & retrospective cohort & 0.62 & $\begin{array}{l}\text { patients from an acute } \\
\text { medical unit }\end{array}$ & CFS & all-cause mortality \\
\hline Kundi H 2020 (34) & Turkey & $74.1 \pm 7.1$ & 18234 & retrospective cohort & 0.47 & hospital patients & Hospital Frailty Risk Score & $\begin{array}{c}\text { all- cause mortality, } \\
\text { COVID-19 severity, } \\
\text { admission to intensive } \\
\text { care unit, application } \\
\text { of invasive mechanical } \\
\text { ventilation, long-length } \\
\text { stay }\end{array}$ \\
\hline Steinmeyer Z 2020 (35) & France & $85.5 \pm 7.5$ & 94 & retrospective cohort & 0.45 & hospital patients & Frail Non-Disabled survey & all-cause mortality \\
\hline Shi SH 2020 (36) & America & $86.8 \pm 9.0$ & 146 & retrospective cohort & 0.35 & long-term care residents & Frailty index & all-cause mortality \\
\hline Hägg S 2020 (37) & Sweden & $81.0 \pm 8.6$ & 250 & retrospective cohort & 0.48 & hospital patients & CFS & all-cause mortality \\
\hline Maguire D 2020 (38) & England & - & 224 & retrospective cohort & 0.55 & hospital patients & CFS & all-cause mortality \\
\hline Ma Y 2020 (46) & China & $60-96$ & 114 & prospective cohort & 0.50 & hospital patients & FRAIL scale & COVID-19 severity \\
\hline Marengoni A 2020 (39) & Italy & $79.5 \pm 6.1$ & 91 & retrospective cohort & 0.60 & hospital patients & CFS & all-cause mortality \\
\hline Darren A 2020 (40) & England & $81.1 \pm 8.1$ & 677 & retrospective cohort & 0.54 & hospital patients & CFS & all-cause mortality \\
\hline Miles A 2020 (47) & England & $80.0 \pm 6.8$ & 217 & prospective cohort & 0.62 & hospital patients & CFS & all-cause mortality \\
\hline Owen RK 2020 (41) & England & $78.8 \pm 8.3$ & 285 & retrospective cohort & 0.54 & hospital patients & CFS & all-cause mortality \\
\hline Fiorentino M 2020 (42) & America & $60.7 \pm 15$ & 374 & retrospective cohort & 0.59 & hospital patients & $\begin{array}{c}\text { Palliative Performance } \\
\text { Scale }\end{array}$ & all-cause mortality \\
\hline Smet RD 2020 (43) & Belgium & $85.0 \pm 6.2$ & 81 & retrospective cohort & 0.41 & hospital patients & CFS & all-cause mortality \\
\hline Burns GP 2020 (44) & England & $81.5 \pm 9.2$ & 28 & retrospective cohort & 0.54 & hospital patients & CFS & all-cause mortality \\
\hline Hewitt J 2020 (26) & England \& Italy & $74 \pm 13.5$ & 1564 & retrospective cohort & 0.58 & hospital patients & CFS & all-cause mortality \\
\hline Rawle MJ 2020 (45) & England & $86.0 \pm 7.6$ & 134 & retrospective cohort & 0.55 & hospital patients & CFS & all-cause mortality \\
\hline
\end{tabular}

CFS: Clinical Frailty Scale

the random-effects meta-analysis was used. Otherwise, the fixed-effects meta-analysis would be used. we conducted the stratified analysis to test interactions according to age(mean or median of age of more than 80 versus mean or median of age of less than 80 ), source of patients(patients from hospitals versus patients from secondary care settings), definition of frailty(clinical frailty score, and other definitions of frailty), the quality of included studies(high quality and low-moderate quality), adjustment method(multivariate versus univariate), and then we conducted meta-regression to explore the effect of these stratified factors on the association between frailty and mortality risk. Besides, The publication bias was evaluated by funnel plot and calculation of the Egger test and the Begg test (33). The sensitivity analysis was performed by excluding included studies one by one.

\section{Results}

\section{Search results}

A total of 581 relevant articles were retrieved in public and gray databases (Figure 1). 268 articles were further screened after the removal of duplicates. According to the titles and abstracts screening, 240 non-relative articles were removed, 73 potentially eligible studies for full-text screening remained (Supplementary Table 1). Finally, sixteen studies were included according to the predefined inclusion and exclusion criteria.
Figure 1

The flowchart of study screening

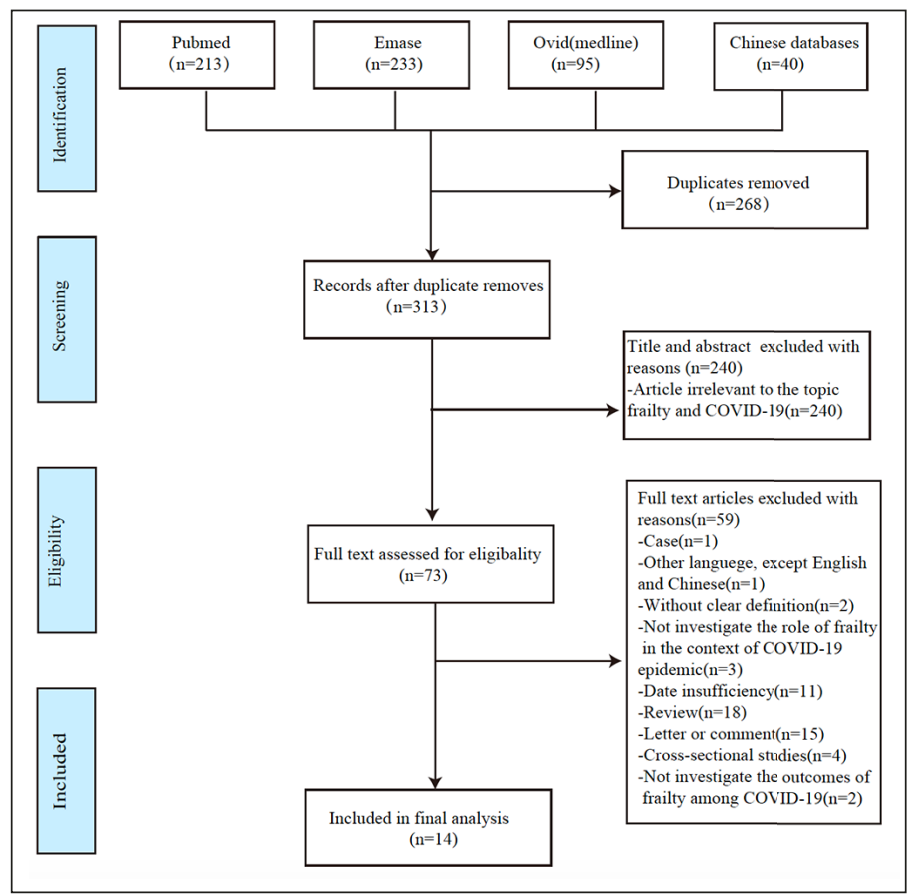


Table 2

Risk of mortality in COVID-19 for frail patients compared with non-frail: stratified analysis with meta-regression

\begin{tabular}{lccc}
\hline Stratified analysis & No. Of studies cohort & Pooled RR(95\%) & Meta-regression P value \\
\hline Mean or median of age & 8 & $2.52(2.32,2.74)$ & 0.435 \\
Less than 80 & 7 & $1.51(1.22,1.87)$ & \\
More than 80 & & & 0.454 \\
Frailty definition & 10 & $1.92(1.51,2.43)$ & $1.63(1.09,2.43)$ \\
Clinical Frailty Scale & 5 & & $1.90(1.41,2.58)$ \\
Other frailty definitions & & $1.73(1.28,2.34)$ & 0.477 \\
Adjustment method & 7 & $1.53(1.41,1.66)$ \\
Multivariate & 8 & $1.85(1.49,2.30)$ \\
Univariate & & \\
Participants source & 2 & $1.77(1.42,2.20)$ \\
Secondary care settings & 13 & $2.09(1.50,2.90)$ & 0.178 \\
Hospital s & &
\end{tabular}

Abbreviation: RR, risk ratio; $\mathrm{CI}$, confidence interval

\section{Included studies characteristics}

The characteristics of the included studies with 4324 patients were shown in Table 1, including fourteen retrospective cohort studies $(26,27,34-45)$ and two prospective cohort studies $(46$, 47). These studies were conducted in regions from America, Europe and Asia. Thirteen studies included patients with a mean or median of age of more than 65 year $(26,27,34-37,40$, $41,43-45)$. Fourteen studies were patients from the hospitals $(26,34,35,37-47)$, one from long-term care (36), and another from an acute medical unit (27). Six kind of measurement tools of frailty were used in the included studies, with clinical frailty score, frailty index, hospital frailty risk score, frail nondisabled survey, FRAIL scale, and palliative performance scale (Supplementary Table 2).

\section{Quality assessment}

The quality of included studies was assessed by Newcastle Ottawa Scale (Supplementary Table 3). Of the sixteen cohort studies, 13 studies had high quality $(\geq 7)[26,27,34,37,38$, $40-47]$ and two studies achieved a max score of $9(26,46)$.

The association of frailty with clinical events among patients with COVID-19

Frailty was reported to be significantly associated with mortality risk among patients with COVID-19 in several studies, and non-survivors had higher clinical frailty score than survivors $(21,23,26,43,48-50)$. In terms of the frailty phenotype parameters, slow walk speed was demonstrated associated with increased an risk of mortality (51). However, Doglietto et al. reported that clinical frailty score was comparable in severe and non-severe groups (52). However, some studies suggested that frailty was not associated with increased adverse outcomes (53). To resolve the contradictions, we conducted a meta-analysis in eleven retrospective cohort studies reporting the association between frailty and the risk of all-cause mortality. As a result, frailty was significantly associated with an increased risk of mortality, with pooled risk ratios of 1.81 (95\% confidence intervals: $1.48,2.21$, $\mathrm{I} 2=87.0 \%, \mathrm{P}<0.001$ ) (Figure 2).We further explored the association between frailty and mortality risk with frailty assessed by clinical frailty score as a continuous variable. We conducted a pooled analysis with four studies, demonstrated clinical frailty score (per additional point) was significantly associated with all-cause mortality, with pooled risk ratios of 1.74 (95\% confidence intervals: $1.40,2.18, \mathrm{I} 2=5 \%, \mathrm{P}<0.001)($ Supplemental Figure 1).

For other clinical events, frailty was significantly associated with an increased risk of COVID-19 severity with pooled risk ratios of 7.47 (95\% confidence intervals:1.73, 32.40, I2=- \%, $\mathrm{P}<0.001$ ), admission to the intensive care unit with pooled risk ratios of 1.80 (95\% confidence intervals: $1.19,2.71, \mathrm{I} 2=96.2 \%$, $\mathrm{P}=0.005)$, application of invasive mechanical ventilation with pooled risk ratios of 1.57 (95\% confidence intervals:1.21,2.03, $\mathrm{I} 2=88.0 \%, \mathrm{P}=0.003)$, long-length stay with pooled risk ratios of 1.22 (95\% confidence intervals: $1.07,1.40, \mathrm{I} 2=73.6 \%, \mathrm{P}=0.003$ ) (Supplementary Table4). 


\section{THE IMPACT OF FRAILTY ON COVID-19 OUTCOMES}

Figure 2

Association of frailty with all-cause mortality among COVID-19

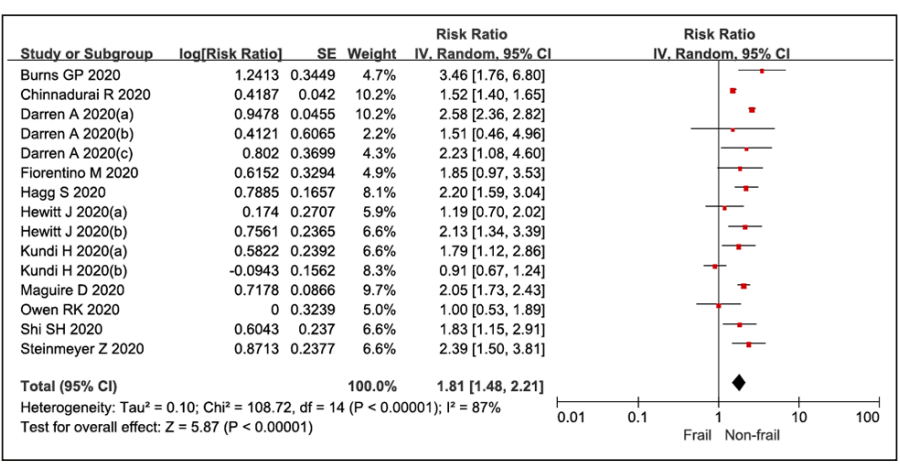

\section{Stratified analyses of included studies and meta-regression}

Stratified analysis was conducted to test the association of frailty with all-cause mortality at different levels (Supplementary Table 5 and Appendix Figure 3-7). According to stratified analyses of age and patients source, frailty was associated with mortality risk with pooled risk ratios of 1.51 (95\% confidence intervals: $1.22,1.87, \mathrm{I} 2=74.0 \%, \mathrm{P}<0.001$ ) for age with mean or median of age of more than 80 year, 2.52 (95\% confidence intervals: $2.32,2.74, \mathrm{I} 2=0 \%, \mathrm{P}<0.001$ ) for age with mean or median of age of less than 80 years, $1.85(95 \%$ confidence intervals: $1.49,2.30, \mathrm{I} 2=80.0 \%, \mathrm{P}<0.001$ ) for patients from hospitals, 1.53 (95\% confidence intervals:1.41,1.66, $\mathrm{I} 2=0 \%, \mathrm{P}<0.001)$ for patients from secondary care settings. Meanwhile, a decline in heterogeneity was observed in the stratified analyses of age and patients source. On the other hand, frailty was still considered as a useful tool for predicting the mortality risk, with stratified analysis of the measurement methods of frailty definition with the pooled risk ratios of 1.92 (95\% confidence intervals: $1.51,2.43, \mathrm{I} 2=89.0 \%, \mathrm{P}<0.001$ ) for clinical frailty score; 1.63 (95\% confidence intervals:1.09,2.43, $\mathrm{I} 2=74 \%, \mathrm{P}<0.001)$ for other frailty definitions; adjustment method with pooled risk ratios of 1.90 (95\% confidence intervals: $1.41,2.58, \mathrm{I} 2=92.0 \%, \mathrm{P}<0.001)$ for multivariate, and 1.73 (95\% confidence intervals: $1.28,2.34, \mathrm{I} 2=76.0 \%, \mathrm{P}<0.001)$ for univariate; study quality with pooled risk ratios of 1.77 (95\% confidence intervals: $1.42,2.20, \mathrm{I} 2=89.0 \%, \mathrm{P}<0.001$ ) for high quality; 2.09 (95\% confidence intervals: 1.50,2.90, $\mathrm{I} 2=0 \%, \mathrm{P}<0.001)$ for low-moderate quality. Furthermore, metaregression found these methodological characteristics did not alter the association between frailty and all-cause mortality.

\section{Sensitivity analysis and publication bias}

In order to investigate the reliability and stability of the association between frailty and all-cause mortality, we performed sensitivity analysis by excluding the included studies in our meta-analysis one by one, with the result that frailty was still associated with an increased risk of all-cause mortality (Supplementary Table 5). Publication bias was assessed by funnel plot and calculation of the Egger test and the Begg test. The value of Egger test and Begg test for the association between frailty and all-cause mortality was 0.739 and 0.843 , respectively, which indicated no publication bias existed.

\section{Discussion}

To our knowledge, this study represents one of the earliest meta-analysis that collected all evidence about the role of frailty in patients with COVID-19, and the pooled analysis demonstrated a high prevalence of frailty among patients with COVID-19. Frailty was significantly associated with an increased risk of adverse clinical outcomes, including all- cause mortality, COVID-19 severity, admission to the intensive care unit, application of invasive mechanical ventilation and longlength stay.

As SARS-CoV-2 is now still spreading worldwide rapidly, many countries paid great attention to medical research about COVID-19, while there is a still a lack of effective drugs to control the diseases (54). However, it is worth mentioning that understanding the prognosis of the disease and scientific management may benefit COVID-19 patients more (55).

Frailty is characterized by increasing vulnerability to stress due to the decline of physiologic reserve in immune, metabolic and neuromuscular systems (56). SARS-COV-2 enters into the body through binding the host cell surface ACE2 receptor bind (57). Meanwhile, ACE2 is ubiquitously expressed in extensive cell types in multiple organs, including lung, heart, kidney and intestine (58-60). Previous studies demonstrated that COVID-19 patients exhibited severe organ-injuries with $14.0 \%$ respiratory distress syndrome, $15.0 \%$ cardiac injury, $15.7 \%$ hepatic injury, and $13.7 \%$ renal injury (60-62). Therefore, patients with COVID-19 may tend to be frail, especially old people. Woolford et al reported COVID-19 positive patients increased 1.4-fold risk of frailty (51).

Through the centralized collection and comprehensive analysis of existing research data, we also demonstrated that frailty could increase the risk of death by 1.81 times in this sudden epidemic, which indicated frailty measurement was an effective tool for identifying the high risk of death among patients infected SARS-COV-2. Meanwhile, frailty assessment in the hospital is a concise and low-cost way to screen and identify a group of patients who were at greater risk of adverse outcomes (63). On the other hand, plder people had a high percentage of death among patients with COVID-19 patients due to multiple organ dysfunction (64-66), Therefore, identification frail older people who were more likely to have adverse clinical outcomes and paying more intensive care for them would contribute to increased survival and recovery rates.

Frail patients were more likely to present with other chronic diseases, potentially increasing COVID-19 mortality rate. Guan et al demonstrated patients with COPD (2.68-fold), diabetes (1.59-fold), hypertension (1.58-fold) and malignancy (3.50fold) were more likely to reach adverse clinical outcomes 


\section{THE JOURNAL OF NUTRITION, HEALTH \& AGING}

than those without comorbidity (67). Meanwhile, patients with at least one comorbidity and those with two or more comorbidities increased 1.79-fold and 2.59-fold risk of composite end-point, respectively (67). Patients with frailty were found to have unbalance in inflammation and immune regulation, such as increased pro-inflammatory bio-markers and peripheral white blood cells $(68,69)$. A meta-analysis of 35 studies demonstrated that frailty and pre-frailty were associated with higher inflammatory parameters, especially C-reactive protein and Interleukin-6 (70). Thus, SARS-CoV-2 might exacerbate the status of inflammation and immune of frail patients, even contributed to forming a cytokines storm, which greatly increased the risk of mortality.

Indeed, previous studies had demonstrated that frailty was associated with an increased risk of falls, disease progression, intensive care unit admission and all-cause mortality (71). For instance, Muscedere et al investigated the impact of frailty in the intensive care unit with the result that the prevalence of frailty was $30 \%$, and frailty was associated with high hospital mortality risk (11). In addition, the International Conference of Frailty and Sarcopenia Research (Iclinical frailty scoreR) recommended screening frailty in the older people by implying a validated setting-specific tools. For individuals who is screened positive for frailty, more comprehensive assessments and interventions would be performed to improve the prognosis of frail patients (52). The latest research, which did not do the quantitative analysis, pointed out that frailty may have contributed to the vulnerability of the older people to more severe clinical presentation during the COVID-19 pandemic, the use of frailty assessment to make the next decision had been recommended clinically (53). Therefore, it is rational to use frailty measurement tools during the pandemic of COVID-19, which could be help make decision.

However, there were contradictory results reported in several studies. Knopp et al. And Miles et al.reported that increased clinical frailty score was not related to the mortality in older patients with $\operatorname{COVID}(28,47)$, while the reliability of the results was limited to its sample source and inadequate adjusting confounds. In addition, Doglietto et al demonstrated that the clinical frailty score score in severe patients with COVID-19 was higher compared with patines who had mild symptoms, although the difference was not significant (72). Nevertheless, the results may be limited by the small sample size in the both mild and severe groups. Rhiannon $\mathrm{KO}$ et al. confirmed that illness severity and comorbidities, but not frailty, slightly increased the risk of dying from COVID-19. However, it had to be mentioned that uneven local service configuration may be an essential factor leading to the results (41).

\section{Limitations}

There were still several limitations. First, the studies included in the final analysis were retrospective observational studies, which could not provide higher strength of evidence than prospective cohort studies. Second, in order to perform a meta-analysis, we converted odds ratios into risk ratios due to differences in association measures and heterogeneity of study designs, leading to inflated risk estimates. Moreover, we conducted a pooled analysis combining crude and adjusted risk ratio estimates, which was still controversial. Third, there were few studies included to analyze the association between frailty and clinical outcomes, except all-cause mortality, which might weaken the reliability of pooled analysis in our study. Fourth, the existence of heterogeneity in the meta-analysis for association between frailty and all-cause mortality. Fortunately, the result of pooled analysis was not changed in the subsequent stratified analysis and sensitivity analysis. Nevertheless, we observed a decline in heterogeneity in stratified analyses with age and patients source. Meanwhile these factors was no identified in meta-regression, which may be explained by lacking statistical power in meta-regression (73). Above all, the result needed to be validated in further well-designed prospective cohort studies.

\section{Conclusion}

This systematic review and meta-analysis demonstrated frailty was significantly associated with an increased risk of clinical adverse events (all- cause mortality, COVID-19 severity, admission to the intensive care unit, application of invasive mechanical ventilation, long-length stay). Given the epidemic of COVID-19 and shortage of medical resources, paying more attention to screen frailty would contribute to disease management and resource allocation among patients with COVID-19.

Acknowledgement: None.

Conflict of interest: None.

Funding source: Funding source from science and technology research project of Jiangxi Provincial Department of Education (GJJ150960), production study research project of Gannan Medical University (cp201407) and «Xinglin scholar» subject talent research plan of Chengdu University of traditional Chinese Medicine(YYZX2019004).

Ethical Standards: None.

\section{References}

1. Pareek M, Bangash MN, Pareek N et al. Ethnicity and COVID-19: an urgent public health research priority. Lancet 2020; 395(10234): 1421-1422.

2. https://covid19.who.int/.

3. Severin R, Arena R, Lavie CJ et al. Respiratory Muscle Performance Screening for Infectious Disease Management Following COVID-19: A Highly Pressurized Situation. Am J Med 2020; 133(9)1025-1032.

4. Zhu Y, Xie J, Huang F et al. Association between short-term exposure to air pollution and COVID-19 infection: Evidence from China. Sci Total Environ 2020; 727: 138704.

5. Brook RD, Levy P, Rajagopalan S . Cardiometabolic Risk Factor Control During Times of Crises and Beyond. Circ Cardiovasc Qual Outcomes 2020;13(7):006815.

6. Brenner EJ, Ungaro RC, Gearry RB et al.Corticosteroids, But Not TNF Antagonists, Are Associated With Adverse COVID-19 Outcomes in Patients With Inflammatory Bowel Diseases: Results From an International Registry. Gastroenterology 2020; 159(2): 481-491.

7. Liang W, Liang H, Ou L et al.Development and Validation of a Clinical Risk Score to Predict the Occurrence of Critical Illness in Hospitalized Patients With COVID-19. JAMA Intern Med 2020; 180(8): 1081-1089.

8. Clegg A, Young J, Iliffe S et al. Frailty in elderly people. Lancet 2013; 381(9868):752762 . 


\section{THE IMPACT OF FRAILTY ON COVID-19 OUTCOMES}

9. Zhou J, Huang P, Liu P et al. Association of vitamin D deficiency and frailty: A systematic review and meta-analysis. Maturitas 2016; 94: 70-76.

10. Kojima G. Frailty as a Predictor of Future Falls Among Community-Dwelling Older People: A Systematic Review and Meta-Analysis. J Am Med Dir Assoc 2015;16(12):1027-1033.

11. Muscedere J, Waters B, Varambally A et al. The impact of frailty on intensive care unit outcomes: a systematic review and meta-analysis. Intensive Care Med. 2017;43(8): 1105-1122.

12. Cunha AIL, Veronese N, de Melo Borges S et al.Frailty as a predictor of adverse outcomes in hospitalized older adults: A systematic review and meta-analysis. Ageing Res Rev 2019;56: 100960 .

13. Kojima G,Iliffe S, Taniguchi Y et al. Prevalence of frailty in Japan: A systematic review and meta-analysis. J Epidemiol 2017;27(8):347-353.

14. Kojima G. Prevalence of Frailty in Nursing Homes: A Systematic Review and MetaAnalysis. J Am Med Dir Assoc; 2015. 16(11): 940-945.

15. Houghton JSM, Nickinson ATO, Morton AJ et al. Frailty Factors and Outcomes in Vascular Surgery Patients: A Systematic Review and Meta-analysis. Ann Surg; 2019.

16. Houghton JSM, Nickinson ATO, Morton AJ et al. Frailty Factors and Outcomes in Vascular Surgery Patients: A Systematic Review and Meta-analysis. Ann Surg 2020; 272(2): 266-276.

17. Shen HQ, Wu B, Xie HP et al. Preparation of Axially Chiral 2,2'-Biimidazole Ligands through Remote Chirality Delivery and Their Application in Asymmetric Carbene Insertion into N-H of Carbazoles. Org Lett 2019;21(8): 2712-2717.

18. Yang X, Lupón J, Vidán MT et al.Impact of Frailty on Mortality and Hospitalization in Chronic Heart Failure: A Systematic Review and Meta-Analysis. J Am Heart Assoc 2018;7(23): e008251.

19. Hewitt J, Long S, Carter B et al. The prevalence of frailty and its association with clinical outcomes in general surgery: a systematic review and meta-analysis. Age Ageing 2018; 47(6): 793-800.

20. Kusumastuti S, Rozing MP, Lund R et al. The added value of health indicators to mortality predictions in old age: A systematic review. Eur J Intern Med 2018; 57: 7-18.

21. Bellelli G, Rebora P, Valsecchi MG et al. Frailty index predicts poor outcome in COVID-19 patients. Intensive Care Med 2020; 46(8): 1634-1636.

22. Tandon P, Tangri N, Thomas L et al. A Rapid Bedside Screen to Predict Unplanned Hospitalization and Death in Outpatients With Cirrhosis: A Prospective Evaluation of the Clinical Frailty Scale. Am J Gastroenterol 2016;111(12): 1759-1767.

23. Labenz C, Kremer WM, Schattenberg JM et al. Clinical Frailty Scale for risk stratification in patients with SARS-CoV-2 infection. J Investig Med 2020; 68(6): 1199-1202.

24. Rockwood K, Song X, MacKnight $\mathrm{C}$ et al. A global clinical measure of fitness and frailty in elderly people. Cmaj 2005;173(5): 489-495.

25. Han SA, KochVG. Clinical and Ethical Considerations in Allocation of Ventilators in an Influenza Pandemic or Other Public Health Disaster: A Comparison of the 2007 and 2015 New York State Ventilator Allocation Guidelines. Disaster Med Public Health Prep 2020;1-10.

26. Hewitt J,Carter B, Vilches-Moraga A et al. The effect of frailty on survival in patients with COVID-19 (COPE): a multicentre, European, observational cohort study. Lancet Public Health 2020; 5(8):444-451.

27. Chinnadurai R, Ogedengbe $\mathrm{O}$, Agarwal $\mathrm{P}$ et al. Older age and frailty are the chief predictors of mortality in COVID-19 patients admitted to an acute medical unit in a secondary care setting- a cohort study. BMC Geriatr 2020;20(1): 409.

28. Knopp P, Miles A, Webb TE et al. Presenting features of COVID-19 in older people: relationships with frailty, inflammation and mortality. Eur Geriatr Med 2020; 1-6.

29. Darvall JN, Bellomo R, Bailey M et al. Frailty and outcomes from pneumonia in critical illness: a population-based cohort study. Br J Anaesth 2020;125(5): 730-738

30. Pan L, Huang P, Xie X et al. Metabolic associated fatty liver disease increases the severity of COVID-19: A meta-analysis. Dig Liver Dis 2020.

31. Willi C, Bodenmann P, Ghali WA et al. Active smoking and the risk of type 2 diabetes: a systematic review and meta-analysis. Jama 2007; 298(22): 2654-2664.

32. Pourbaix A, Lafont Rapnouil B, Guéry R et al. Smoking as a Risk Factor of Invasive Fungal Disease: Systematic Review and Meta-Analysis. Clin Infect Dis 2020; 71(4): 1106-1119.

33. Zhang Y, Fang F, Tang $\mathbf{J}$ et al. Association between vitamin D supplementation and mortality: systematic review and meta-analysis. Bmj 2019;366: 14673.

34. Kundi H, Çetin EhÖ, Canpolat U et al. The role of Frailty on Adverse Outcomes Among Older Patients with COVID-19. J Infect 2020.

35. Steinmeyer Z, Vienne-Noyes S, Bernard M et al. Acute Care of Older Patients with COVID-19: Clinical Characteristics and Outcomes. Geriatrics (Basel) 2020; 5(4).

36. Shi SM,Bakaev I,Chen H et al. Risk Factors, Presentation, and Course of Coronavirus Disease 2019 in a Large, Academic Long-Term Care Facility. J Am Med Dir Assoc 2020; 21(10): 1378-1383.

37. Hägg S, Jylhävä J, Wang, Y et al. Age, Frailty, and Comorbidity as Prognostic Factors for Short-Term Outcomes in Patients With Coronavirus Disease 2019 in Geriatric Care. J Am Med Dir Assoc 2020.

38. Maguire D, Woods $\mathrm{M}$, Richards $\mathrm{C}$ et al. Prognostic factors in patients admitted to an urban teaching hospital with COVID-19 infection. J Transl Med 2020; 18(1): 354.

39. Marengoni A, Zucchelli A, Grande G et al. The impact of delirium on outcomes for older adults hospitalised with COVID-19. Age Ageing 2020.

40. Aw D, Woodrow L, Ogliari G et al. Association of Frailty with Mortality in Older Inpatients with Covid-19: A Cohort Study. Age Ageing 2020.

41. Owen RK, Conroy SP, Taub $\mathrm{N}$ et al. Comparing associations between frailty and mortality in hospitalised older adults with or without COVID-19 infection: a retrospective observational study using electronic health records. Age Ageing 2020.

42. Fiorentino M, Pentakota SR, Mosenthal AC et al. The Palliative Performance Scale predicts mortality in hospitalized patients with COVID-19. Palliat Med 2020;34(9) 1228-1234.

43. De Smet R, Mellaerts B, Vandewinckele H et al. Frailty and Mortality in Hospitalized Older Adults With COVID-19: Retrospective Observational Study. J Am Med Dir Assoc 2020;21(7): 928-932.

44. Burns GP, Lane ND, Tedd HM et al. Improved survival following ward-based noninvasive pressure support for severe hypoxia in a cohort of frail patients with COVID19: retrospective analysis from a UK teaching hospital. BMJ Open Respir Res 2020 7(1).

45. Rawle MJ, Bertfield DL, Brill SE. Atypical presentations of COVID-19 in care home residents presenting to secondary care: A UK single centre study. Aging Medicine 2020 .

46. Ma Y, Hou L, Yang X et al. The association between frailty and severe disease among COVID-19 patients aged over 60 years in China: a prospective cohort study. BMC Med 2020;18(1):274.

47. Miles A, Webb TE, McLoughlin BC et al. Outcomes from COVID-19 across the range of frailty: excess mortality in fitter older people. Eur Geriatr Med 2020;11(5):851-855.

48. Brill SE, Jarvis HC, Ozcan E et al. COVID-19: a retrospective cohort study with focus on the over-80s and hospital-onset disease. BMC Med 2020;18(1):194

49. Hoek RAS, Manintveld OC, Betjes MGH et al. COVID-19 in solid organ transplant recipients: a single-center experience. Transpl Int 2020.

50. Knights H, Mayor N, Millar K et al. Characteristics and outcomes of patients with COVID-19 at a district general hospital in Surrey, UK. Clin Med (Lond) 2020;20(5):148-153.

51. Woolford SJ, D'Angelo S, Curtis EM et al. COVID-19 and associations with frailty and multimorbidity: a prospective analysis of UK Biobank participants. Aging Clin Exp Res 2020;32(9):1897-1905.

52. Dent E, Morley JE, Cruz-Jentoft AJ et al. Physical Frailty: ICFSR International Clinical Practice Guidelines for Identification and Management. J Nutr Health Aging 2019; 23(9):771-787.

53. Maltese G,Corsonello A, Di Rosa M et al. Frailty and COVID-19: A Systematic Scoping Review. J Clin Med 2020;9(7).

54. Xu L, Mao Y, Chen G. Risk factors for 2019 novel coronavirus disease (COVID-19) patients progressing to critical illness: a systematic review and meta-analysis. Aging (Albany NY) 2020; 12(12): 12410-12421

55. Qin JJ, Cheng X, Zhou F et al. Redefining Cardiac Biomarkers in Predicting Mortality of Inpatients With COVID-19. Hypertension 2020;76(4): 1104-1112.

56. Crapo RO, Kanner RE, Jensen RL et al. Variability of the single-breath carbon monoxide transfer factor as a function of inspired oxygen pressure. Eur Respir J 1988;1(6): 573-574.

57. Hoffmann M, Kleine-Weber H, Schroeder S et al. SARS-CoV-2 Cell Entry Depends on ACE2 and TMPRSS2 and Is Blocked by a Clinically Proven Protease Inhibitor Cell 2020; 181(2): 271-280.

58. Tipnis SR,Hooper NM,Hyde R et al. A human homolog of angiotensinconverting enzyme. Cloning and functional expression as a captopril-insensitive carboxypeptidase. J Biol Chem 2000; 275(43):33238-33243.

59. Velavan TP, Meyer CG. The COVID-19 epidemic. Trop Med Int Health 2020; 25(3): 278-280.

60. Potere N, Valeriani E, Candeloro $\mathrm{M}$ et al. Acute complications and mortality in hospitalized patients with coronavirus disease 2019: a systematic review and metaanalysis. Crit Care 2020; 24(1):389.

61. Ahmed J, Rizwan T, Malik F et al. COVID-19 and Liver Injury: A Systematic Review and Meta-Analysis. Cureus 2020;12(7): 9424

62. Yang $\mathrm{X}$, Jin $\mathrm{Y}, \mathrm{Li} \mathrm{R}$ et al. Prevalence and impact of acute renal impairment on COVID-19: a systematic review and meta-analysis. Crit Care 2020;24(1): 356.

63. Gilbert T, Neuburger J, Kraindler J et al. Development and validation of a Hospita Frailty Risk Score focusing on older people in acute care settings using electronic hospital records: an observational study. Lancet 2018; 391(10132): 1775-1782.

64. Liu PP, Blet A, Smyth D et al. The Science Underlying COVID-19: Implications for the Cardiovascular System. Circulation 2020; 142(1):68-78 


\section{THE JOURNAL OF NUTRITION, HEALTH \& AGING}

65. Guo T, Shen Q, Guo W et al. Clinical Characteristics of Elderly Patients with COVID19 in Hunan Province, China: A Multicenter, Retrospective Study. Gerontology 2020; 66(5): 467-475.

66. Zhao M, Wang M, Zhang $\mathrm{J}$ et al. Comparison of clinical characteristics and outcomes of patients with coronavirus disease 2019 at different ages. Aging (Albany NY) 2020; 12(11):10070-10086.

67. Guan W.J, Liang WH, Zhao Y et al. Comorbidity and its impact on 1590 patients with COVID-19 in China: a nationwide analysis. Eur Respir J 2020; 55(5).

68. Pansarasa O, Pistono C, Davin A et al. Altered immune system in frailty: Genetics and diet may influence inflammation. Ageing Res Rev 2019; 54: 100935

69. Ferrucci L, Fabbri E. Inflammageing: chronic inflammation in ageing, cardiovascular disease, and frailty. Nat Rev Cardiol 2018;15(9):505-522.
70. Soysal P, Stubbs B, Lucato P et al. Inflammation and frailty in the elderly: A systematic review and meta-analysis. Ageing Res Rev 2016; 31:1-8.

71. Dent E, Martin FC, Bergman H et al. Management of frailty: opportunities, challenges, and future directions. Lancet 2019; 394(10206): 1376-1386.

72. Doglietto F, Vezzoli M, Gheza F et al. Factors Associated With Surgical Mortality and Complications Among Patients With and Without Coronavirus Disease 2019 (COVID19) in Italy. JAMA Surg 2020; 155(8): 1-14.

73. Hardy RJ, Thompson SG. Detecting and describing heterogeneity in meta-analysis. Stat Med 1998; 17(8): 841-856. 